\title{
Photoconductivity in macroporous silicon with regular structure of macropores
}

\author{
V.I. Ivanov, L.A. Karachevtseva, N.I. Karas, O.A. Lytvynenko, \\ K.A. Parshin, and A.V. Sachenko \\ V. Lashkaryov Institute of Semiconductor Physics, 45, prospect Nauky, 03028 Kyiv, Ukraine \\ Phone: 525 9815, fax: 525 8243, e-mail: lakar@isp.kiev.ua
}

\begin{abstract}
The effects of the increase of photoconductivity in periodic macroporous silicon structures depending on the size and period of cylindrical macropores are investigated. It is obtained that the ratio of macroporous silicon photoconductivity to bulk silicon photoconductivity achieves a maximum at the distance between macropores equal to two thicknesses of the Schottky layer, which corresponds to the experimental data. The increase of photoconductivity is due to both the large total surface area of macropores and the existence of Schottky layers in the near-surface region of cylindrical macropores.
\end{abstract}

Keywords: macroporous silicon, photoconductivity, Schottky layer.

Manuscript received 04.05.07; accepted for publication 19.12.07; published online 13.02.08.

\section{Introduction}

Two-dimensional photonic structures based on macroporous silicon are perspective for their use in the infrared region due to an increase of the optical absorption [1], a high photoconductivity in the intrinsic absorption region [2], and the presence of additional bands of photoconductivity [3]. The existence of deep macropores periodically arranged in the n-type silicon matrix provides a large effective surface area (Fig. 1), which determines the optical and electrophysical properties of structures. Increasing the light absorption in macroporous silicon structures by two orders of magnitude with respect to that of bulk silicon was measured for the wavelengths shorter than the optical period of a structure [1]. The dependence of photoconductivity on the incidence angle and the light absorption higher than the reflection in macroporous silicon are explained by the interaction of optical modes with surface oscillators on the macropore surface and the generation of optical polaritons [2]. The absolute maximum of photoconductivity is measured at the distance between macropores equal to $2 \mu \mathrm{m}$. The dependences of conductivity, concentration, and mobility of electrons on the sizes and the concentration of macropores were measured on a two-layer structure "macroporous silicon-silicon" [4]. The obtained results were explained by the formation of a near-surface region around macropores with a thickness of $1 \mu \mathrm{m}$ after the electrochemical and chemical etching. It was established that the microstructure of a macropore surface and the built-in field depend on parameters of the electrochemical process such as voltage and current density [5]. The value of the built-in field on a macropore surface is defined by the surface concentration of $\mathrm{Si}-\mathrm{O}$ and $\mathrm{Si}-\mathrm{H}$ bonds [6]. In addition, the sign of the main maximum in the spectra of electroreflectance and the dependence of its magnitude on the applied constant bias correspond to the formation of inversion layers (Schottky layers) on the macropore surface. In the present article, we will determine the conductivity and photoconductivity of macroporous silicon structures for various sizes and periods of cylindrical macropores, by taking the Schottky layers in the silicon matrix into account.

\section{Methodology}

The starting material consisted of n-type silicon with orientation [100] and $4.5-\mathrm{Ohm} \cdot \mathrm{cm}$ resistivity $\left(n_{0}=\right.$ $10^{15} \mathrm{~cm}^{-1}$ ). The periodic structures were anisotropically etched in a $10 \%$ solution of $\mathrm{KOH}$ after the photolithography procedure with formation of periodic pits. Macropores were formed due to the generation and transfer of nonequilibrium holes to the electrochemically treated surface of $\mathrm{n}-\mathrm{Si}$ as a result of the backside optical band-to-band electron-hole generation [7, 8]. According to the optical microscope study, macropores were formed with diameters $D_{p}=1-10 \mu \mathrm{m}$ and periods $a=$ $3.5-14 \mu \mathrm{m}$.

\section{(C) 2007, V. Lashkaryov Institute of Semiconductor Physics, National Academy of Sciences of Ukraine}




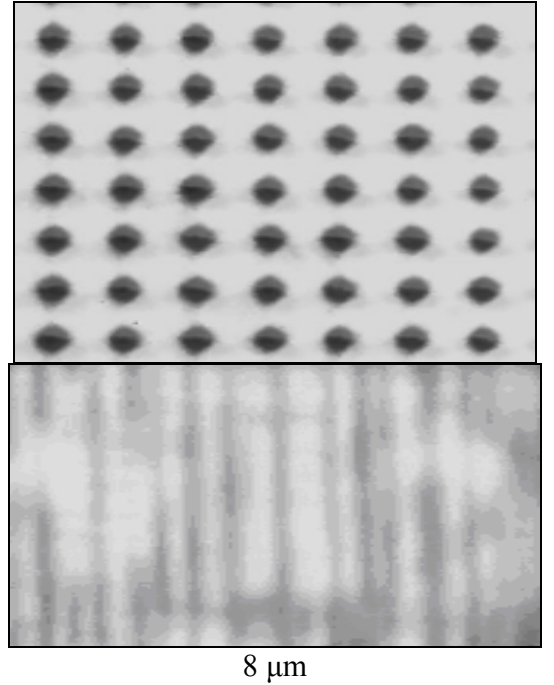

Fig. 1. Optical microscope photo of a two-dimensional macroporous silicon photonic structure with cylindrical macropores of $4 \mu \mathrm{m}$ in period and $1.8 \mu \mathrm{m}$ in diameter.

The In/monocrystal $\mathrm{n}-\mathrm{Si}$ and $\mathrm{In} /$ macroporous $\mathrm{n}-\mathrm{Si}$ contacts were formed by thermal evaporation of indium in the atmosphere of hydrogen in the same 4-probe configuration at the 4-mm distance between contacts with a transient resistance of $4-10 \mathrm{Ohm} \cdot \mathrm{cm}^{2}$. The electrical parameters (conductivity, charge carrier concentration, and mobility) were obtained by the 4probe method at $300 \mathrm{~K}$. The photoconductivity spectra were measured at the normal light incidence on the macroporous silicon structure with screening contacts at light wavelengths $\lambda=0.8-1.5 \mu \mathrm{m}$ by using a spectrometer IRS-31. Photoconductivity of macroporous silicon structures as a function of the illumination intensity was measured at $0.95 \mu \mathrm{m}$ using a light diode 3L130A.

\section{Parameters of the inversion potential on the macropore surface}

For the macroporous silicon structures under study, we calculated the dependence of the dimensionless surface potential on the surface level concentration (Fig. 2). There are the inversion potentials for surface level concentrations between $10^{11}$ and $10^{12} \mathrm{~cm}^{-2}$. For surface level concentrations less than $10^{11} \mathrm{~cm}^{-2}$, the inversion potential transforms to a depleted one and sharply diminishes with a reduction of the surface level concentration.

The thickness of a Schottky layer on the macropore surface is determined by the formula [9]

$w=0.182\left(\frac{T}{300} \frac{10^{15}}{n_{0}}\right)^{0.5} \sqrt{2 \ln \left(\frac{n_{0}}{n_{i}(T)}\right)}$,

where $T$ is the absolute temperature, $n_{0}$ and $n_{i}(T)$ are the equilibrium electron concentration in a silicon matrix and the intrinsic charge carrier concentration in silicon, respectively. Fig. 3 shows the theoretical dependences of the Schottky layer thickness on temperature according to (1). The observed decrease of the Schottky layer thickness with increase in temperature is due to a decrease of the intrinsic charge carrier concentration. Thus, for the investigated macroporous silicon structures, the Schottky layer thicknesses are equal to $w=0.9-1.15 \mu \mathrm{m}$ at $77-300 \mathrm{~K}\left(n_{0}=10^{15} \mathrm{~cm}^{-1}\right)$ and determine the dark resistance of the silicon matrix for the structures with the distance between macropores $a-$ $D_{p} \approx 0.6-3.6 \mu \mathrm{m}$.

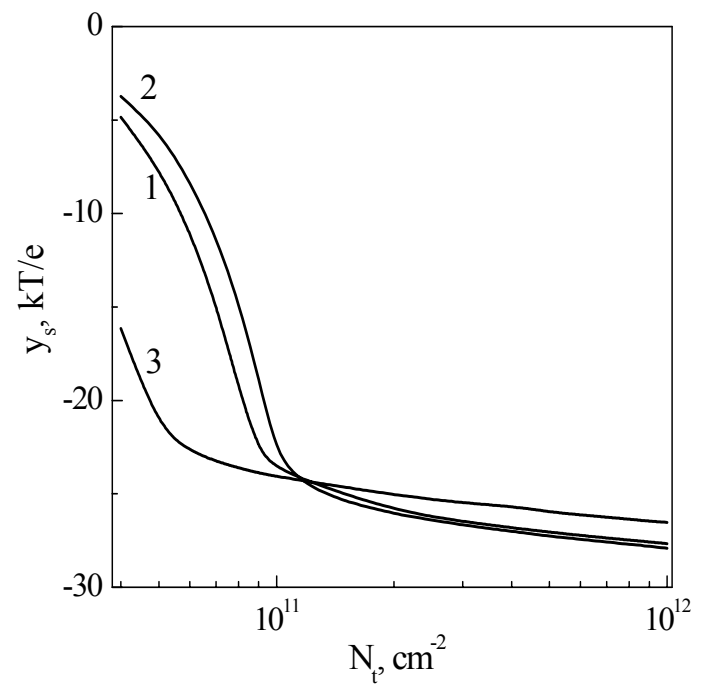

Fig. 2. Dependence of the surface potential in silicon on the surface level concentration at room temperature $\left(n_{0}=\right.$ $10^{15} \mathrm{~cm}^{-3}$ ). Values of the parameter $n_{0}$ are as follows: $1-10^{15}$, $2-3 \cdot 10^{15}, 3-3 \cdot 10^{14} \mathrm{~cm}^{-3}$.

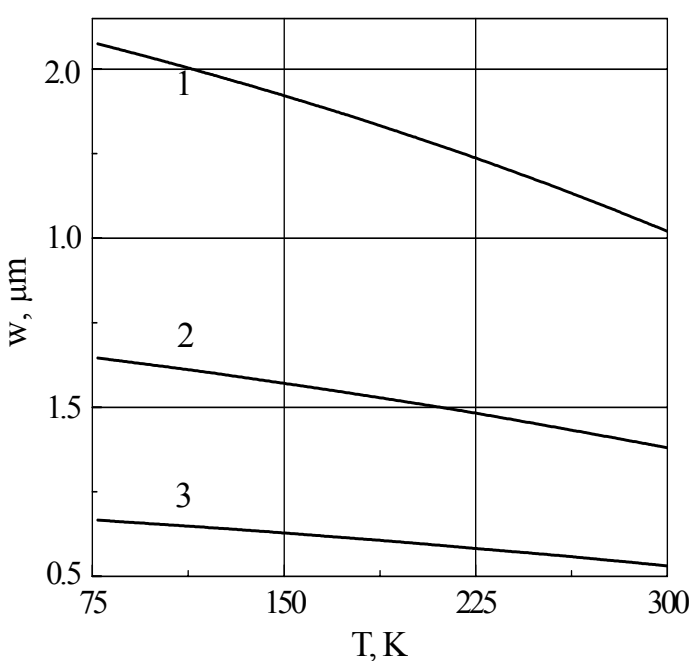

Fig. 3. Dependence of the Schottky layer thickness (in $\mu \mathrm{m}$ ) on temperature. Values of the parameter $n_{0}$ are as follows: 1 $3 \cdot 10^{14}, 2-10^{15}, 3-3 \cdot 10^{15} \mathrm{~cm}^{-3}$. 


\section{Dependence of conductivity on the geometry of pores in macroporous silicon structures}

To find the dark conductivity and photoconductivity, we consider macroporous silicon with a regular structure of cylinder macropores with period $a$. The elementary cell consists of four adjacent cells with the macropore diameter $D_{p}$ and the distance between pores $a-D_{p}$ (Fig. 4). The ratio of the area of macropores to that of the crystal surface is equal to

$\frac{S_{p}}{S_{0}}=\frac{\pi\left(D_{p}+2 w\right)^{2}}{2 a^{2}}$,

taking the Schottky layers of thickness $w$ around a macropore into account. Here, $S_{0}$ is the area of the elementary cell surface limited to lines between the centers of macropores, and $S_{p}$ is the part of $S_{0}$ occupied by macropores.

The dark resistance of a crystal with regular structure of cylinder macropores was determined as two series resistances of the silicon matrix (1) with area $S_{0}-S_{p}$ and length $h$, and (2) with area $S_{0}$ and length $d-h$. This gives

$$
R_{d p}=R_{0} \frac{d-h}{d}\left(1+\frac{h}{d-h} \frac{S_{0}}{S_{0}-S_{p}}\right),
$$

where $R_{0}$ is the dark resistance of a crystal without pores. Using (1), we obtain

$$
R_{d p}=R_{0} \frac{d-h}{d}\left(1+\frac{h}{d-h} \frac{1}{1-\frac{\pi\left(D_{p}+2 \cdot w\right)^{2}}{2 \cdot a^{2}}}\right) .
$$

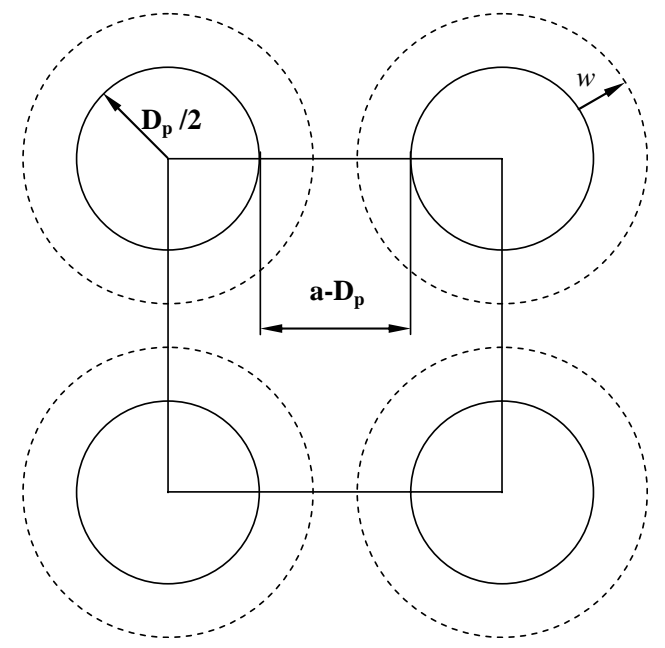

Fig. 4. Elementary cell including 4 neighboring pores: $D_{p} / 2-$ the pore radius, $a-D_{p}-$ distance between pores, $w-$ Schottky layer thickness.

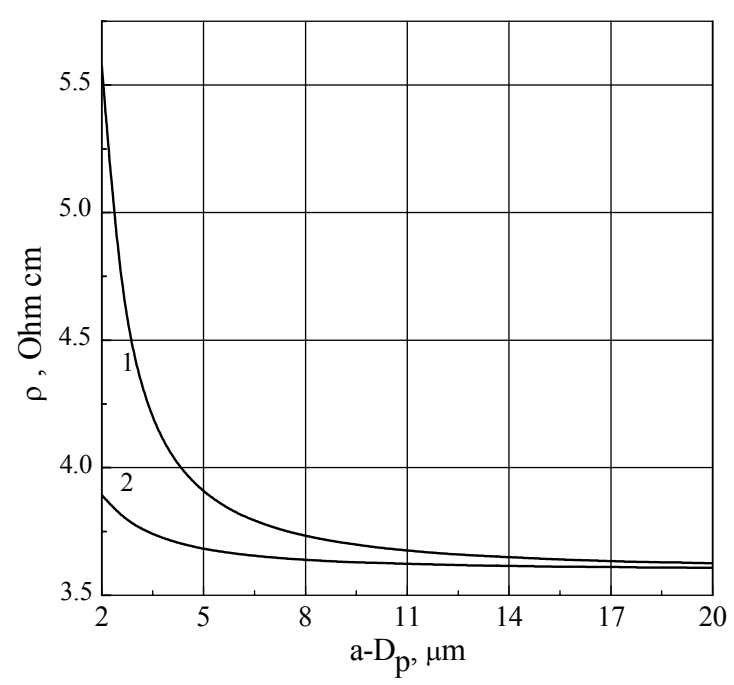

Fig. 5. Dependence of the macroporous silicon resistance on the distance between pores for the injection level of $1.3 \cdot 10^{15} \mathrm{~cm}^{-3}$ and the Schottky layer thicknesses: $1-0.78 \mu \mathrm{m}, 2-0 \mu \mathrm{m}$. The used parameters are $D_{p}=2 \mu \mathrm{m}, h=100 \mu \mathrm{m}$, and $d=300 \mu \mathrm{m}$.

Dependences of the dark resistance on the distance between macropores are shown in Fig. 5 in the case where the pore diameter is equal to $2 \mu \mathrm{m}$. Curve 1 corresponds to the Schottky layer thickness $w=1 \mu \mathrm{m}$, while curve 2 corresponds to the absence of Schottky layers. The resistance of the crystal increases in the presence of macropores more rapidly, when there are the Schottky layers around pores with $w=D_{p} / 2$. The last result corresponds to the experimental data for macroporous silicon structures for $a-D_{p} \approx 2.5 \mu \mathrm{m}$ close to the results of theoretical calculations.

\section{Dependence of photoconductivity on the pore geometry in macroporous silicon structures}

Under the estimated earlier conditions, the ratio of the photoconductivities of a macroporous silicon structure with highly depleted or inversed surface potential and the bulk silicon is proportional to the ratio of the total pore surface area $S_{s p}$ to the structure surface area $S_{0}$ :

$\frac{S_{s p}}{S_{0}}=\frac{\pi h D_{p}}{a^{2}}$

at $a-D_{p} \geq 2 w$. This ratio attains the value of 50-100 for the macroporous silicon structures under study. When the distance between macropores is less than the double thickness of a Schottky layer $\left(a-D_{p}<2 w\right)$, the resulted depleted layer becomes thinner, and the ratio of the photoconductivities of macroporous silicon, $\sigma_{\mathrm{ph} p}$, and bulk silicon, $\sigma_{\mathrm{ph} b}$, diminishes

$\frac{\sigma_{\mathrm{ph} p}}{\sigma_{\mathrm{ph} b}} \approx\left\{\begin{array}{l}\frac{\pi h D_{p}}{a^{2}}, \text { for } a-D_{p} \geq 2 w \\ \frac{\pi h D_{p}}{a^{2}} \frac{a-D_{p}}{2 w}, \text { for } a-D_{p}<2 w\end{array}\right.$ 


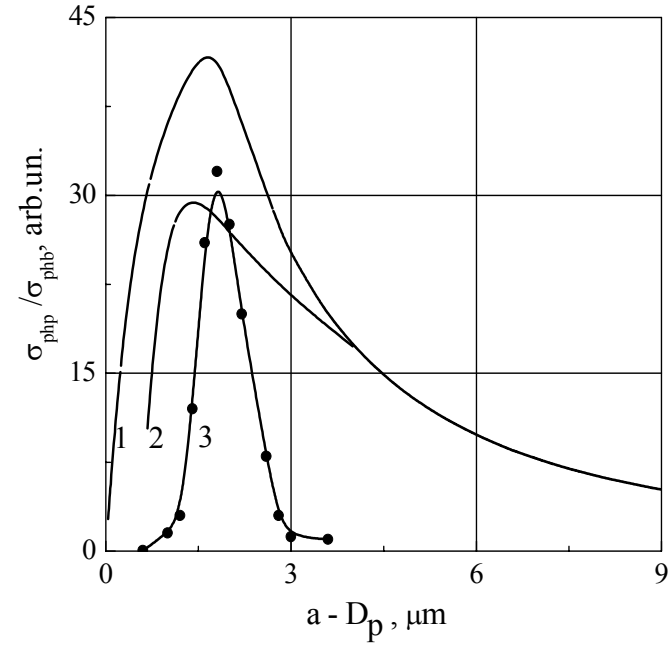

Fig. 6. Ratio of the photoconductivities of macroporous silicon and bulk silicon versus the distance between pores: 1 theoretical calculations for $D_{p}=2 \mu \mathrm{m}, n_{0}=10^{15} \mathrm{~cm}^{-3} ; 2-$ experimental data for a macroporous silicon structure with $D_{p}=$ $0.5-6 \mu \mathrm{m}, a-D_{p}=0.65-4 \mu \mathrm{m}, n_{0}=10^{15} ; 3-$ the results of theoretical calculations and experimental data.

The sharp decrease of this ratio is due to the overlapping of Schottky layers and a decrease of the conductivity modulation under illumination. The dependences of ratio (6) on the distance between pores $a-D_{p}$ are shown in Fig. 6 with a maximum value of 44 at $a-D_{p}=2 w$. It should be noted that expression (6) is valid when macroporous silicon becomes excited uniformly along the full depth of macropores. The experimentally measured maximum value of the ratio of photoconductivities is 32 at $a-D_{p} \approx 2 \mu \mathrm{m}$ (Fig. 6) for a silicon optical absorption depth of $50 \mu \mathrm{m}$ (the absorption coefficient is $2 \cdot 10^{2} \mathrm{~cm}^{-1}$ for $\left.\lambda=0.95 \mu \mathrm{m}\right)$ that corresponds to the double thickness of a Schottky layer. The experimental data are in good agreement with the results of theoretical calculations.

According to the examined model, the main contribution to the macroporous silicon photoconductivity is made by illumination-induced modulations of the conductivity of Schottky layers. Here, the Schottky layer thickness is less than that in darkness that corresponds to the additional electron conductivity. In the case of conductivity inversion, the Gibbs excess of electrons under illumination is given by

$$
\begin{aligned}
& \Delta N=0.182 \cdot 10^{-4}\left(\frac{T}{300} \frac{10^{15}}{n_{0}}\right)^{0.5} \times \\
& \times\left[\sqrt{2 \ln \left(\frac{n_{0}}{n_{i}(T)}\right)}-\sqrt{2 \ln \left(\frac{n_{0}}{n_{i}(T)}\right)-\Delta y_{s}},\right.
\end{aligned}
$$

where $\Delta y_{s}$ is a change of the surface potential under illumination. The value of $\Delta y_{s}$ is found from the electroneutrality equation that includes a surface level charge, a non-equilibrium hole charge, and a charge of completely ionized donors in a Schottky layer under illumination. The surface states were approximated by two discrete surface levels [9]. Thus, the ratio of the photoconductivity of macroporous silicon and the dark conductivity of bulk silicon is determined by the formula

$\sigma_{p h} / \sigma_{d p}=q \mu_{n} \kappa \frac{\Delta N}{w} \frac{\pi h D_{p}}{a^{2}} \frac{a-D_{p}}{2 w} R_{0}$,

where $\mu_{n}$ is the electron mobility and $\kappa$ is a coefficient considerably less than unity which takes into account the fact that Schottky layers do not fill completely the space between pores. The ratio $\sigma_{\mathrm{ph}} / \sigma_{d p}$ logarithmically depends on $\Delta n$, and the photosensitivity of a crystal with pores is larger than the bulk silicon photosensitivity. The dependence of $\Delta n$ on the illumination intensity $I$ is given by relation

$$
\Delta n=\frac{\beta I}{S+D / L},
$$

where $\beta$ is the quantum efficiency, $S$ is the effective surface recombination velocity on pores, and $D$ and $L$ are the coefficient and the length of diffusion of nonequilibrium charge carriers, respectively.

In the case of the inversion or depleted potential, the effective surface recombination velocity can be low [9], and its value has tendency to the reduction when the injection level increases. Thus, the dependence of $\Delta n$ on the illumination intensity can be either linear or ultra linear. For $D \approx 10 \mathrm{~cm}^{2} / \mathrm{s}$ and $L \approx 10^{-2} \mathrm{~cm}$, the value of $\Delta n$ is proportional to $I$ with the proportionality coefficient of about $10^{-3}$. The dependence of the photoconductivity of a silicon crystal with regular pores on the illumination intensity is shown in Fig. 7. In this case, the macroporous silicon photoconductivity can attain up to $10 \%$ of the dark conductivity at the light intensities $10^{13}-10^{15}$ photon $/\left(\mathrm{cm}^{2} \mathrm{~s}\right)$ that is ten times larger than the bulk silicon photoconductivity. As can be seen from (5), such an increase of the photosensitivity is due to both the large total surface area of macropores and the presence of the Schottky layers around pores.

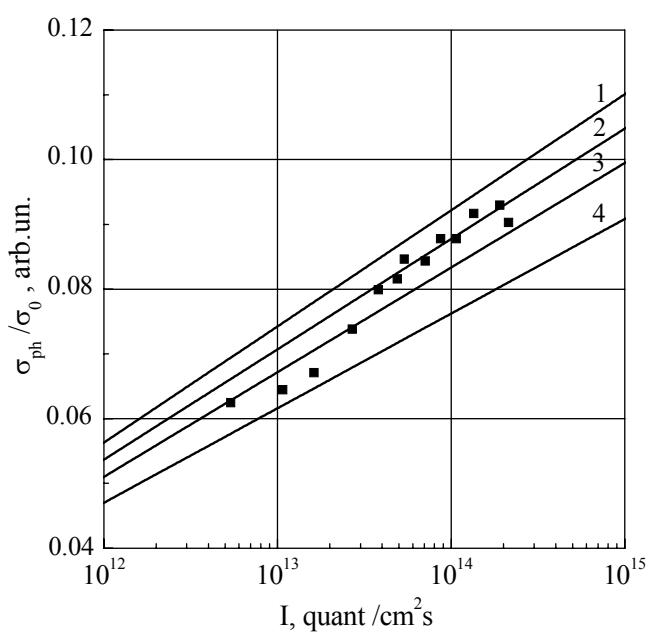

Fig. 7. Ratio of the photoconductivity of macroporous silicon to the dark conductivity versus the illumination intensity. The used values of $a-D_{p}, \mu \mathrm{m}$ : are: $1-2,2-3,3-1,4-5$. The experimental data $\left(a-D_{p}\right.$ equals $\left.2 \mu \mathrm{m}\right)$ are marked by dots. 


\section{Conclusions}

The increase of the photoconductivity is due to the large total surface area of macropores and the presence of Schottky layers in the near-surface region of cylindrical macropores.

In the present work, we have investigated the effects of an increase of the photoconductivity in periodic structures of macroporous silicon depending on a size of cylindrical macropores and the inversion of conductivity on the pore - silicon interface (Schottky layers). The ratio of the photoconductivities of macroporous silicon and bulk silicon attains a maximum at the distance between macropores equal to the double thickness of a Schottky layer that corresponds to the experimental data. It is shown that the sharp decrease of this ratio with decrease in the distance between pores is due to the overlapping of Schottky layers and a decrease of the conductivity modulation under illumination. The calculated and experimental values of the photoconductivity of macroporous silicon can attain $10 \%$ of the dark conductivity at an illumination intensity of $10^{13}-10^{15}$ quantum $\mathrm{cm}^{-2} \mathrm{~s}^{-1}$ that is by one order of magnitude greater than that in bulk silicon. The increase of the photosensitivity is due to the large total surface area of macropores and the presence of Schottky layers in the near-surface region of cylindrical macropores.

\section{References}

1. L.A. Karachevtseva, O.A. Lytvynenko, E.A. Malovichko, E.J. Stronska, Optical transmittance of 2D macroporous silicon structures // Semiconductor Physics, Quantum Electronics \& Optoelectronics 4(1), p. 347-351 (2001).
2. L.A. Karachevtseva, N.I. Karas', V.F. Onischenko, F.F. Sizov, Surface polaritons in 2D macroporous silicon structures // Nanotechnology 3(1), p. 76-88 (2006).

3. L. Karachevtseva, A. Glushko, M. Karas, V. Onischenko, Surface waves in 2D photonic macroporous silicon structures // Proc. SPIE 5733, p. 297-304 (2005).

4. L.A. Karachevtseva, O.A. Lytvynenko, E.A. Malovichko, V.D. Sobolev, and O.L. Stronska, Electrical properties of macroporous silicon structures // Semiconductor Physics, Quantum Electronics \& Optoelectronics 4(1), p. 40-48 (2001).

5. R.Yu. Holiney, L.A. Matveeva, E.F. Venger, L.A. Karachevtseva, O.A. Lytvynenko, Electroreflectance study of macroporous silicon surface // Appl. Surf. Sci. 172, p. 214-219 (2001).

6. L.A. Karachevtseva, O.A. Litvinenko, E.I. Stronskaya, Investigation of the local chemical states in the structures of macroporous silicon // Theoretical and Experimental Chemistry 39(2), p. 85-89 (2003).

7. L.A. Karachevtseva, O.A. Litvinenko, E.A. Malovichko, Stabilization of electrochemical formation of macropores in $n$-Si // J. Theor. and Experim. Chem. 34(5), p. 314-318 (1998).

8. L.A. Karachevtseva, O.A. Litvinenko, E.I. Stronska, Development and optical characteristics of the macroporous silicon structures // Semiconductor Physics, Quantum Electronics \& Optoelectronics, 3(1), p. 22-26 (2000).

9. A.V. Sachenko, O.V. Snitko, Photoeffects in NearSurface Semiconductor Layers. Naukova Dumka, Kyiv, 1984 (in Russian). 